\title{
Tailoring hole spin splitting and polarization in nanowires
}

\author{
D. Csontos \\ Institute of Fundamental Sciences, Massey University, Private Bag 11 222, Palmerston North, New Zealand \\ U. Zülicke \\ Institute of Fundamental Sciences and MacDiarmid Institute for Advanced Materials and Nanotechnology, \\ Massey University, Private Bag 11 222, Palmerston North, New Zealand and \\ Institut für Theoretische Festkörperphysik and DFG Center for Functional \\ Nanostructures (CFN), Universität Karlsruhe, D-76128 Karlsruhe, Germany
}

\begin{abstract}
Spin splitting in $p$-type semiconductor nanowires is strongly affected by the interplay between quantum confinement and spin-orbit coupling in the valence band. The latter's particular importance is revealed in our systematic theoretical study presented here, which has mapped the range of spin-orbit coupling strengths realized in typical semiconductors. Large controllable variations of the $g$-factor with associated characteristic spin polarization are shown to exist for nanowire subband edges, which therefore turn out to be a versatile laboratory for investigating the complex spin properties exhibited by quantum-confined holes.
\end{abstract}

Engineering spin splitting of charge carriers in semiconductor nanostructures may open up intriguing possibilities for realizing spin-based electronics $\frac{1}{\underline{1}}$ and quantum information processing. 2 . Due to the generally strong dependence of $g$-factors on band structure, $\underline{\underline{3}}$ it is expected that spatial confinement will have an important effect on Zeeman splitting when bound-state quantization energies are no longer negligible compared with the separation of bulk-material energy bands. The degeneracy of heavy-hole (HH) and light-hole (LH) bulk dispersions at the zone center makes the spin properties of valence-band states especially susceptible to such confinement engineering. ${ }^{4,5,6.7}$ Recent advances in fabrication technology $8,9,10,11,12,13,14,15,16$ have created opportunities to investigate hole spin physics in semiconductor nanowires made from a range of different materials.

In contrast to previous theoretical work ${ }^{17,18,19,20}$ on hole spin splitting in quantum wires, we focus here on the influence of the spin-orbit coupling strength on Zeeman splitting of wire-subband edges. A suitable parameter $\gamma$ quantifying spin-orbit coupling in the valence band can be defined in terms of the effective masses $m_{\mathrm{HH}}$ and $m_{\mathrm{LH}}$ associated with the $\mathrm{HH}$ and $\mathrm{LH}$ bands, 21 respectively: $2 \gamma=$ $\left(m_{\mathrm{HH}}-m_{\mathrm{LH}}\right) /\left(m_{\mathrm{HH}}+m_{\mathrm{LH}}\right)$. Table $\prod$ lists values for $\gamma$ in common semiconductors and states its relation to basic bandstructure parameters $\frac{22}{2}$ A large part of the theoretically possible range $0 \leq \gamma \leq 1 / 2$ is covered by available materials, $\stackrel{23}{, ~}$ enabling a detailed study of the interplay between spin-orbit coupling in the valence band and nanowire confinement. Our

TABLE I: Relative spin-orbit coupling strength $\gamma=\gamma_{s} / \gamma_{1}$ in the valence band of common semiconductors. Here $\gamma_{s}=\left(2 \gamma_{2}+3 \gamma_{3}\right) / 5$, and $\gamma_{1,2,3}$ denote the Luttinger parameters. ${ }^{22}$

\begin{tabular}{cccccc}
\hline \hline ZnTe/ZnS & AlAs/AlP & AlSb & CdTe & GaN/AlN GaAs/InP \\
$0.28^{a}$ & $0.31^{b}$ & $0.32^{b}$ & $0.34^{a}$ & $0.36^{b}$ & $0.37^{b}$ \\
\hline $\mathrm{Ge}$ & $\mathrm{InN}$ & $\mathrm{GaSb}$ & $\mathrm{InAs}$ & $\mathrm{InSb}$ & $\mathrm{GaP}$ \\
$0.38^{a}$ & $0.40^{b}$ & $0.41^{b}$ & $0.45^{b}$ & $0.46^{b}$ & $0.48^{b}$ \\
\hline \hline
\end{tabular}

${ }^{a}$ From Ref. 24

${ }^{b}$ From Ref. 25 theoretical investigation reveals surprising qualitative differences in the hole spin properties of nanowires depending on the value of $\gamma$, showing that spin splitting (and polarization) of zone-center valence-band edges in nanowires is highly tunable and has a complex materials dependence. A detailed understanding of these properties is vital for proper interpretation of optical and transport measurements as well as for the design of spintronic applications involving $p$-doped semiconductor nanowires.

We use the Luttinger model ${ }^{22}$ in the spherical approximation $^{26}$ for the top-most bulk valence bands. Including the bulk Zeeman term $H_{\mathrm{Z}}=-2 \kappa \mu_{\mathrm{B}} B \hat{J}_{z}$, the Hamiltonian is given by

$$
H=-\frac{\gamma_{1}}{2 m_{0}} p^{2}+\frac{\gamma_{s}}{m_{0}}\left[(\mathbf{p} \cdot \hat{\mathbf{J}})^{2}-\frac{5}{4} p^{2} \mathbf{1}_{4 \times 4}\right]+H_{\mathrm{Z}} \quad .
$$

Here $\mathbf{p}$ is the linear orbital momentum, $\hat{\mathbf{J}}$ the vector of spin$3 / 2$ matrices, $m_{0}$ the electron mass in vacuum, $\gamma_{s}=\left(2 \gamma_{2}+\right.$ $\left.3 \gamma_{3}\right) / 5$ in terms of the Luttinger parameters, ${ }^{22} \mu_{\mathrm{B}}$ is the Bohr magneton and $\kappa$ the bulk hole $g$-factor. We neglect the small anisotropic part of the bulk-hole Zeeman splitting. A hardwall confinement in the $x y$ plane defines the quantum wire with either cylindrical or square cross-section. Our method for finding the zone-center subband edges and calculating their $g$-factor $g^{*}$ in a magnetic field parallel to the wire axis has been described elsewhere ${ }^{20.27}$ An intriguing universal behavior of wire-subband spin splittings emerges when the bulkZeeman term dominates the orbital effects which, in principle, also contribute to the effective $g$-factor. This universal regime, which is characterized by $g^{*}$ scaling with $\kappa$ and being independent of wire diameter, is accessible in real nanowire systems ${ }^{10}$ where $\kappa$ is enhanced by the $p$ - $d$ exchange interaction with magnetic acceptor ions ${ }^{24}$ Figure 11 illustrates that, for the highest (i.e., closest to the top of the valence band) GaAs hole-wire levels, only a moderate enhancement of $\kappa$ is needed to quench orbital contributions to the $g$-factor. Similar results are obtained for other materials. In the following, we focus entirely on the properties of hole-wire subband-edge $g$ factors in the universal regime where orbital contributions can be neglected.

Our results are summarized in Figure 2 where we show $g$ - 


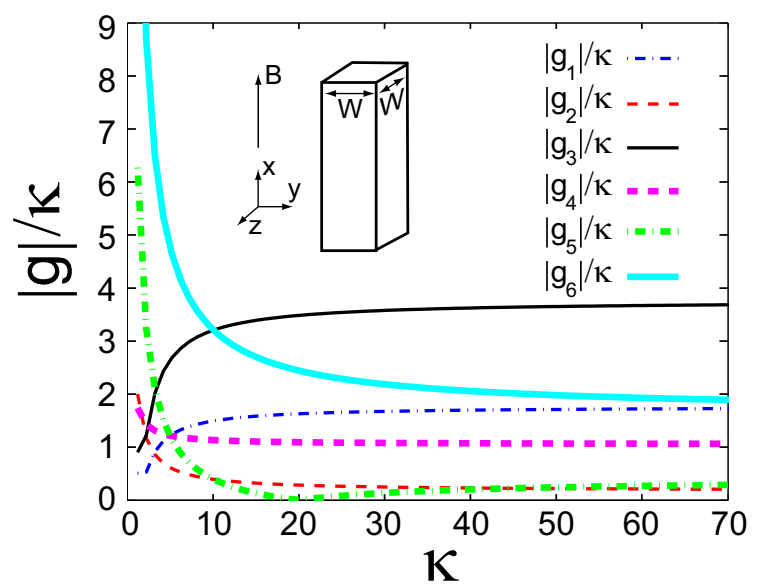

FIG. 1: (Color online) Effective $g$-factors for the six highest zonecenter subband edges in a GaAs wire with square cross-section, plotted as a function of the bulk-hole $g$-factor $\kappa$. An order of magnitude enhancement in $\kappa$ leads to saturation, in effect quenching orbital contributions to the Zeeman splitting.

factors for the ten highest zone-center subband edges in cylindrical hole nanowires, calculated for various spin-orbit coupling strengths $\gamma$. A naïve assumption that the hole spin projection parallel to the wire axis should be quantized would lead us to expect to find only two possible values for the $g$-factor; namely $6 \kappa$ and $2 \kappa$ for the HH and LH states, respectively. Evidently, our results are quite different. Firstly, for any given material, the $g$-factor values vary strongly between the different wire-subband edges, some levels even displaying vanishing $g$-factors. Such seemingly random fluctuations can be explained ${ }^{20,27}$ by nontrivial microscopic hole spin-polarization profiles of wire-subband bound states. Large $g$-factors are found for subband edges with predominantly $\mathrm{HH}$ or LH character, whereas subbands with mixed HH-LH char-

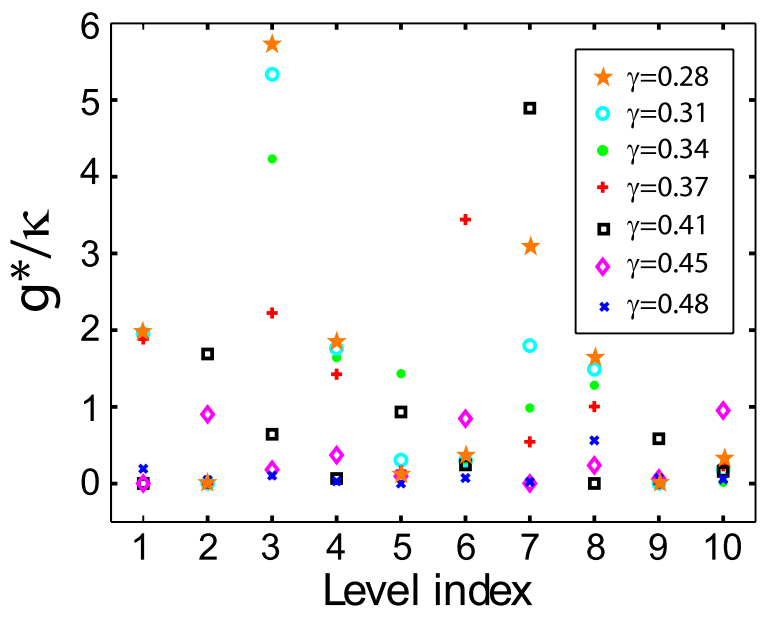

FIG. 2: (Color online) Effective $g$-factors for the ten highest zonecenter subband edges in cylindrical hole nanowires, calculated for various spin-orbit coupling strengths. acter or with vanishing hole-spin polarization have strongly suppressed $g$-factors. We will see below that the intrinsic connection between hole spin splittings and polarizations holds for all materials considered. Secondly, focusing on individual wire levels, it is found that their $g$-factor can vary substantially between different materials. For some subbands, e.g., the third and seventh, the $g$-factors span almost the entire range of values between 0 and $6 \kappa$. For other subbands, $g$-factors cluster around certain values, as is the case of the first, sixth, and tenth levels. Yet other subbands display a seemingly random sequence of alternatingly increasing and decreasing values of $g^{*}$ as the relative spin-orbit coupling strength $\gamma$ is varied.

The anomalous spin splittings in hole nanowires can be attributed to strong HH-LH mixing that is present even at the wire-subband edges. The relative spin-orbit coupling strength $\gamma$ determines this mixing. To be able to characterize the spin properties of individual subband-edge bound states independent of any particular spin-projection basis, we utilize scalar invariants of the spin-3/2 density matrix. See Refs. 20.28 for details of the formalism. In particular, we consider the radial variation of the normalized hole-spin dipole density, denoted by $\rho_{1}^{2} / \rho_{0}^{2}$, which provides a measure of the local hole spin polarization. A uniform value of $\rho_{1}^{2} / \rho_{0}^{2}=9 / 5(1 / 5)$ indicates a $\mathrm{HH}(\mathrm{LH})$ state characterized by a $\hat{J}_{z}$-projection quantum number $\pm 3 / 2( \pm 1 / 2)$. As previously discussed, Zeeman splitting for such a state in a magnetic field parallel to the $z$ axis arises with effective $g$-factor $6 \kappa(2 \kappa)^{22}$ Figure 3 shows

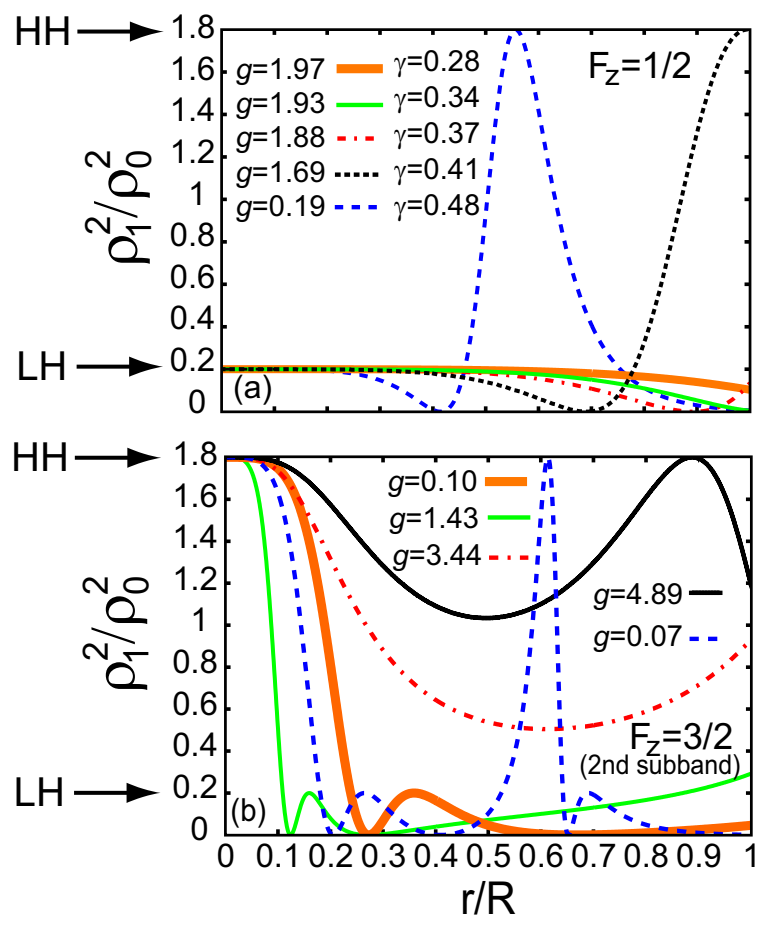

FIG. 3: (Color online) Squared normalized spin-3/2 dipole (spinpolarization) density, $\rho_{1}^{2}(r) / \rho_{0}^{2}(r)$, for (a) the highest subband with $F_{z}=1 / 2$, and (b) the second-highest subband with $F_{z}=3 / 2$. The values of spin-orbit coupling parameter $\gamma$ and corresponding $g$-factor $g \equiv g^{*} / \kappa$ are indicated. 


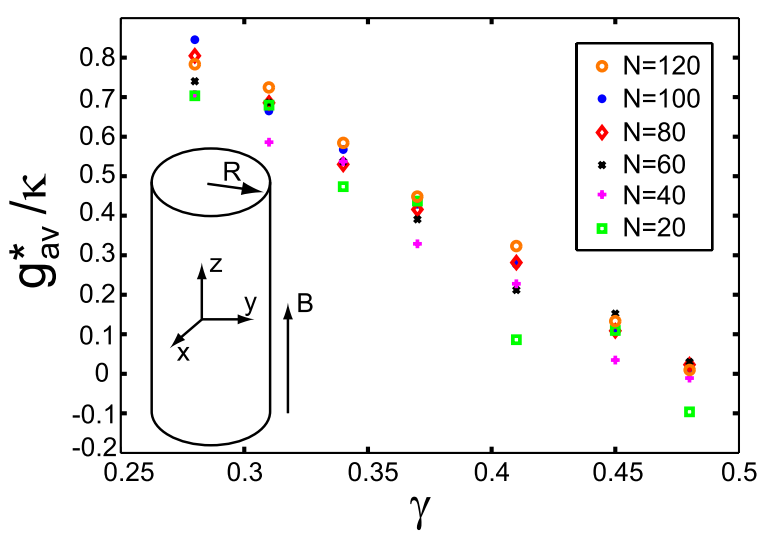

FIG. 4: (Color online) Mean g-factors $g_{\mathrm{av}}^{*}=\frac{1}{N} \sum_{i=1}^{N} g_{i}^{*}$, obtained by averaging over the $N$ highest wire levels, plotted as a function of relative spin-orbit coupling strength $\gamma$. Inset: Wire geometry and orientation of the magnetic field.

the radial spin-polarization profiles $\rho_{1}^{2}(r) / \rho_{0}^{2}(r)$, for the highest hole-wire subband edges with (a) $F_{z}=1 / 2$, and (b) the second-highest subbands with $F_{z}=3 / 2$, for different representative values of $0.28 \leq \gamma \leq 0.48$. Here, $F_{z}$ is the eigenvalue of $\hat{J}_{z}+\hat{L}_{z}$, i.e., the sum of the $z$ components of spin and orbital angular momentum, which is the good quantum number labelling wire-subband bound states ${ }^{20,29}$ Deviations of the hole-spin polarization from the values $9 / 5$ and $1 / 5$ is an indication of the, in principle, ever-present HH-LH mixing in hole wires.

Interestingly, states with $F_{z}=1 / 2$ that form the highest subband edge in systems with $\gamma \leq 0.37$ are quite close to a pure LH character, having $\rho_{1}^{2}(r) / \rho_{0}^{2}(r) \approx 0.2$ across most of the wire radius. However, a continuously increasing trend to develop a HH-LH texture is exhibited for larger $\gamma$. As can be seen, this feature is concomitant with a drastic reduction of the $g$-factor from its value close to $2 \kappa$ that is expected for pure
LH states. A related trend is exhibited by the highest subband edges with $F_{z}=3 / 2$ (not shown here) where, for small values of $\gamma$, the normalized dipole moment is close to the value $9 / 5$ corresponding to a pure $\mathrm{HH}$ state. With increasing $\gamma$, however, the dipole moment is increasingly suppressed. The $g$-factors show a corresponding monotonous suppression, from values close to $6 \kappa$ to values close to 0 .

In contrast to the previous two examples, a very nonmonotonous behavior as a function of $\gamma$ is observed for the second-highest subband edge with $F_{z}=3 / 2$. See Fig. 3 (b) where, for small $\gamma$-values, suppressed polarization profiles correlate with very small effective $g$-factors. As $\gamma$ is increased, the spin dipole moment of the state increases dramatically, approaching values associated with $\mathrm{HH}$ character. [See the dashed-dotted and dashed curves corresponding to $\gamma=0.37,0.41$ in Fig. 3 (b).] The corresponding $g^{*}$ values come close to $6 \kappa$. For yet higher values of $\gamma$, the polarization is again suppressed, with concomitantly vanishing $g$-factors.

A general comparison of polarization profiles for various subband edges with their $g$-factors shows that, as the holespin dipole moment vanishes and/or HH-LH mixing in the radial profile increases, $g^{*}$ is increasingly suppressed. Thus, a direct correlation emerges between the relative spin-orbit coupling strength $\gamma$, the hole-spin polarization, and the Zeeman spin splitting. However, on average, the hole-spin polarization and effective $g$-factors decrease as the relative spin-orbit coupling strength $\gamma$ is increased. This is illustrated by the calculated mean $g$-factors shown in Fig. 4 Such mean values will describe Zeeman splitting in experimental situations where single wire subbands are not resolved. Extrapolating to $\gamma=0.38$, which corresponds to Ge, the value found is consistent with the hole $g$-factor measured recently $\mathrm{y}^{30}$ in rod-shaped quantum dots fabricated from $\mathrm{Ge} / \mathrm{Si}$ core-shell nanowires.

DC acknowledges support from the Massey University Research Fund. The authors benefited from useful discussions with P. Brusheim, A. Führer, S. Roddaro, and H.Q. Xu.
${ }^{1}$ S. A. Wolf, D. D. Awschalom, R. A. Buhrmann, J. M. Daughton, S. von Molnár, M. L. Roukes, A. Y. Chtchelkanova, and D. M. Treger, Science 294, 1488 (2001).

2 D. D. Awschalom, D. Loss, and N. Samarth, eds., Semiconductor Spintronics and Quantum Computation (Springer, Berlin, 2002).

${ }^{3}$ L. M. Roth, B. Lax, and S. Zwerdling, Phys. Rev. 114, 90 (1959).

${ }^{4}$ R. Winkler, S. J. Papadakis, E. P. De Poortere, and M. Shayegan, Phys. Rev. Lett. 85, 4574 (2000).

5 R. Danneau, O. Klochan, W. R. Clarke, L. H. Ho, A. P. Micolich, A. R. Hamilton, M. Y. Simmons, M. Pepper, D. Ritchie, and U. Zülicke, Phys. Rev. Lett. 97, 026403 (2006).

${ }^{6}$ C. E. Pryor and M. E. Flatté, Phys. Rev. Lett. 96, 026804 (2006).

7 K.-M. Haendel, R. Winkler, U. Denker, O. G. Schmidt, and R. J. Haug, Phys. Rev. Lett. 96, 086403 (2006).

${ }^{8}$ L. Samuelson, Mater. Today 6(10), 22 (2003).

9 W. Lu and C. M. Lieber, J. Phys. D: Appl. Phys. 39, R387 (2006).

${ }^{10}$ F. Martelli, S. Rubini, M. Piccin, G. Bais, F. Jabeen, S. De Franceschi, V. Grillo, E. Carlino, F. D'Acapito, F. Boscherini, S. Cabrini, M. Lazzarino, L. Businaro, F. Ro- manato, and A. Franciosi, Nano Lett. 6, 2130 (2006).

11 E. Janik, J. Sadowski, P. Dłużewski, S. Kret, L. T. Baczewski, A. Petroutchik, E. Łusakowska, J. Wróbel, W. Zaleszczyk, G. Karczewski, T. Wojtowicz, and A. Presz, Appl. Phys. Lett. 89, 133114 (2006).

12 J. Johansson, L. S. Karlsson, C. P. T. Svensson, T. Mårtensson, B. A. Wacaser, K. Deppert, L. Samuelson, and W. Seifert, Nat. Mater. 5, 574 (2006).

${ }^{13}$ K. A. Dick, K. Deppert, L. Samuelson, and W. Seifert, J. Cryst. Growth 298, 631 (2007).

${ }^{14}$ L. N. Pfeiffer, R. de Picciotto, K. W. West, K. W. Baldwin, and C. H. L. Quay, Appl. Phys. Lett. 87, 073111 (2005).

15 R. Danneau, W. R. Clarke, O. Klochan, A. P. Micolich, A. R. Hamilton, M. Y. Simmons, M. Pepper, and D. A. Ritchie, Appl. Phys. Lett. 88, 012107 (2006).

16 O. Klochan, W. R. Clarke, R. Danneau, A. P. Micolich, L. H. Ho, A. R. Hamilton, K. Muraki, and Y. Hirayama, Appl. Phys. Lett. 89, 092105 (2006).

17 F. V. Kyrychenko and J. Kossut, Phys. Rev. B 61, 4449 (2000). 
18 Y. Harada, T. Kita, O. Wada, and H. Ando, Phys. Rev. B 74, 245323 (2006).

19 X. W. Zhang and J. B. Xia, J. Phys. D: Appl. Phys. 40, 541 (2007); Eur. Phys. J. B 58, 263 (2007).

${ }^{20}$ D. Csontos and U. Zülicke, Phys. Rev. B 76, 073313 (2007).

${ }^{21}$ In general, the $\mathrm{HH}$ and $\mathrm{LH}$ effective masses have a slight dependence on the direction of wave vector $\mathbf{k}$. We neglect this, typically very small, band warping in the following.

22 J. M. Luttinger, Phys. Rev. 102, 1030 (1956).

${ }^{23}$ In the following, we present results for $0.28 \leq \gamma \leq 0.48$ because nanowires have recently been grown from $\mathrm{ZnTe}^{\overline{11}}(\gamma=0.28)$, $\operatorname{GaAs}^{10}(\gamma=0.37), \mathrm{Ge}^{30}(\gamma=0.38), \operatorname{InAs}^{\frac{13}{3}}(\gamma=0.45)$, and $\mathrm{GaP}^{12}(\gamma=0.48)$.
24 T. Dietl, H. Ohno, and F. Matsukura, Phys. Rev. B 63, 195205 (2001).

25 I. Vurgaftman, J. R. Meyer, and L. R. Ram-Mohan, J. Appl. Phys. 89, 5815 (2001).

${ }^{26}$ N. O. Lipari and A. Baldereschi, Phys. Rev. Lett. 25, 1660 (1970).

27 D. Csontos and U. Zülicke, Physica E, in press (doi: 10.1016/j.physe.2007.09.100).

${ }^{28}$ R. Winkler, Phys. Rev. B 70, 125301 (2004).

29 P. C. Sercel and K. J. Vahala, Appl. Phys. Lett. 57, 545 (1990).

${ }^{30}$ S. Roddaro, A. Fuhrer, C. Fasth, L. Samuelson, J. Xiang, and C. M. Lieber, arXiv:0706.2883 (unpublished). 